\title{
Las percepciones sociales y el consumo de esteroides anabólicos en usuarios de gimnasios en la ciudad de Armenia, 2019
}

\author{
John Steven García Coca'; Isabela García Serna²; Dulfary Mejía Vanegas³; \\ Ángela María Rincon ${ }^{4}$
}

RESUMEN Introducción: esta investigación tiene por objeto estudiar los diferentes factores implicados en las percepciones sociales, que determinan el consumo de esteroides anabólicos en la población adulta en edades de 18 a 40 años que realizan entrenamiento físico, en usuarios de gimnasios en la ciudad de Armenia (Colombia), año 2019.

Método: se describirán los elementos causales por los cuales en los últimos años se ha destacado el desarrollo de un concepto que conlleva tener una imagen perfecta, la cual busca impactar de manera positiva ante toda una sociedad, y comprobar así qué tanto es derivado de la presión social que ejercen los medios, los cuales involucran todos los estratos socioeconómicos de la población, y se acerca cada día más a un problema de salud pública, por las diferentes complicaciones y patologías que desencadena el deseo de una buena imagen corporal desde la percepción social.

Anabolizantes;

PALABRAS Autoimagen;

CLAVE Corticoesteroides;

Percepción Social. 


\section{Social perceptions and anabolic steroid use in gymnasium users in the city of Armenia, 2019}

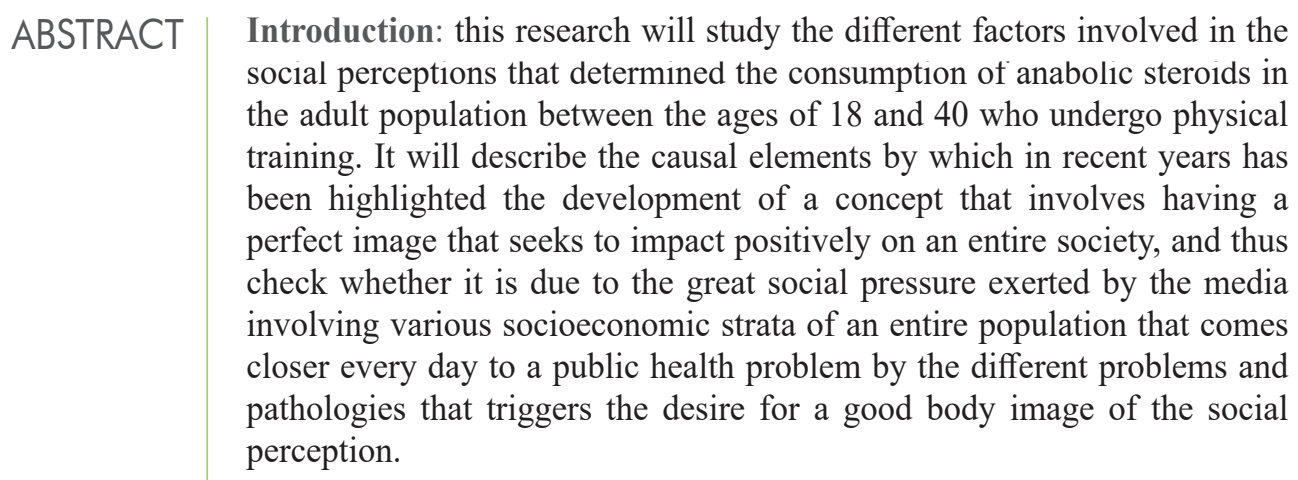

Method: observational, descriptive cross-sectional study, with a population and sample of 70 users between 18 and 40 years of age attending gymnasiums in the city of Armenia, 2019, the analysis techniques used were Tabulation in Excel 2018, SPSS 25. 0 and univariate and bivariate descriptive analysis. for data collection was used a Liker type instrument consisting of 26 questions which evaluate 4 components that are: sociodemographic questions, anthropometric measures, the adonis complex, consumption of anabolic steroids

Expected Results: $100 \%$ of the target population are in an average age of 26 years of age belonging to the health and education sector, which claims to have consumed anabolic steroids to improve their body image from their social perceptions according to the Adonis complex, even knowing that they are dangerous to health, receiving advice from their trainer.

Anabolics;

Self-image;

Corticosteroids;

Social Perception. 


\section{INTRODUCCIÓN}

El consumo de esteroides anabólicos se identifica a partir del inicio de la adultez (desde los 18 años de edad en todos los géneros que actualmente existen). La falta de conocimiento y el afán de querer alcanzar una imagen perfecta, se convierten en una de las tantas causas que conllevan a utilizar y depender de estas sustancias, que sin el debido asesoramiento implica desarrollar alteraciones en las percepciones sociales y trastornos psicológicos que afectan directamente, no solo al individuo sino también a la sociedad, ya que representan efectos negativos que son revelados a corto plazo e involucran conductas nocivas para la sociedad. En conjunto, lo expuesto argumenta la necesidad del compromiso de la sociedad en general, para la prevención y educación en el consumo de esteroides anabólicos, ya que la preocupación por mejorar la imagen corporal se ha convertido en un fenómeno social y un problema de salud pública. Un claro ejemplo de todo ello, es la creciente preocupación por el control de peso que involucra el aumento de masa muscular en los hombres y desarrollar delgadez exagerada en las mujeres, lo que se ve agravado en nuestros días por la influencia que ejercen los medios de comunicación sobre la población (Revuelta, Alonso, Tomás, Guerrero y Rohlfsc, 2004).

Esta problemática incluye enfermedades psicológicas tales como la vigorexia, que hace que los hombres se vean más delgados y débiles, pese a que otras personas los observen grandes y musculosos, y la dismorfia en las mujeres, con un efecto donde ellas se ven gordas y flácidas, cuando realmente los demás las encuentran delgadas y tonificadas.

La importancia del consumo de esteroides anabólicos lo proporciona cada persona desde su idea de imagen corporal y los conceptos que tenga sobre la misma; estas personas se vuelven más susceptibles a la crítica social, y adquieren mayor posibilidad de padecer en un futuro enfermedades, ya sean cardiovasculares, respiratorias, renales, neurológicas y deficiencia reproductiva,

Los esteroides se caracterizan por cumplir diversas funciones en el cuerpo humano: regular el metabolismo, mantener el equilibrio de los electrolitos y la homeostasis, que normaliza los niveles de agua en el cuerpo (4). También benefician la recuperación muscular y aumentan el nivel de masa muscular, utilizado como método el fisicoculturismo. Consumidos en forma inadecuada, pueden llevar a diversas patologías como enfermedades cardiovasculares, neurológicas, respiratorias, hepáticas, renales, disfunción sexual y atrofia muscular (2).

Los esteroides a través de la historia han tenido gran presencia en los estudios clínicos para los avances tecnológicos necesarios en la guerra, con el fin de crear soldados con morfología perfecta y así obtener buenos resultados a la hora de combatir. Fueron descubiertos en 1939 por el alemán Adolf Butenandt obtenidos de las hormonas sexuales masculinas. Adelantos científicos demostraron que había una hormona más fuerte a la hora de sintetizar estos productos, conocida como testosterona, ganando así el más alto reconocimiento a nivel mundial, que lo acredita como uno de los descubrimientos más innovadores de la época (4). 


\section{METODOLOGÍA}

Estudio observacional, descriptivo de corte transversal, con una población y muestra de 100 usuarios entre los 18 y 40 años de edad que asistan a gimnasios de la ciudad de Armenia, 2019. Las técnicas de análisis utilizadas fueron la tabulación en Excel 2018, SPSS 25.0 y análisis descriptivo univariado y bivariado. Para la recolección de datos se utilizó un Instrumento tipo Liker que consta de 26 preguntas, las cuales evalúan 4 componentes: preguntas socio demográficas, medidas antropométricas, el complejo de adonis y consumo de esteroides anabólicos.

\section{RESULTADOS ESPERADOS}

Los usuarios de esteroides anabólicos entre 18 a 40 años, son una respuesta a la imagen corporal con relación a las percepciones sociales que marcan un nuevo concepto de autoimagen. La población adulta femenina percibe su autoimagen corporal de extrema delgadez, mientras que la población masculina en la percepción de la imagen corporal identifica un parámetro de vigorexia, dado por la hipertrofia muscular y la distribución del porcentaje corporal de masa magra.

En el área de la Terapia Respiratoria desde la interdisciplinariedad con los profesionales en Fisiatría, Nutrición, Fisioterapia y Psicología, se divulgarán técnicas fundamentadas en la corporalidad de la respiración, con el fin de mejorar tanto el aspecto físico como emocional, sin la necesidad de utilizar sustancias tóxicas como los esteroides anabólicos, que originan complicaciones en nuestra salud sistémica.

\section{IMPACTOS}

Impacto social: se establecerá la importancia en la Atención Primaria en Salud, de profesionales interdisciplinarios como el Terapeuta Respiratorio, Psicólogo, Nutriólogo y Fisioterapeuta, a través de Programas de Promoción y Prevención en Estilos de Vida Saludable, que concienticen a la población en el consumo de esteroides anabólicos para reducir el riesgo de enfermedades sistémicas.

Impacto económico: esta investigación se ajusta a las Políticas en Salud Pública asociadas a los costos de atención en salud, debido a los factores de riesgo que genera el consumo de esteroides anabólicos, disminuyendo efectos adversos y evitando el uso de terapias de alto costo como la hemodiálisis por falla renal, consecuencia de suplementación tipo hormonal (Esteroides Androgénicos).

Impacto ambiental: los investigadores de este proyecto se comprometen a cuidar al medio ambiente mediante la utilización de papel reciclable, medios magnéticos para la ejecución y desarrollo del proyecto, disminuyendo la contaminación con el plástico, cartón, etc 
REFERENCIAS 1. Arbinaga, F. (2011). Físico-culturistas consumidores de esteroides anabolizantes y sus relaciones con la autodescripción física y la ansiedad física social. Universitas Psychologica, 10 (1), 137-147.

2. Corrientes LACDE. Estudio descriptivo del consumo de esteroides anabólicos en la población que asiste a gimnasios de la ciudad de corrientes, argentina. 2013;1-6.

3. Salabarria K, Rodríguez S, Cruz S. Percepción de la imagen corporal. Osasunaz. 2007; 8:171-183.

4. Avella RE, Medellín JP. Los Esteroides Anabolizantes Androgénicos, Riesgos Y Consecuencias Anabolic Androgenic Steroids, Risks and Consequences. Cient Supl Olimp. 2012; $15: 47-55$.

5. Latorre Román P, Garrido Ruiz A, García Pinillos F. Versión española del cuestionario del complejo de Adonis; un cuestionario para el análisis del dimorfismo muscular o vigorexia. Nutrición Hospitalaria. 2015;31(3):1246-1253. 


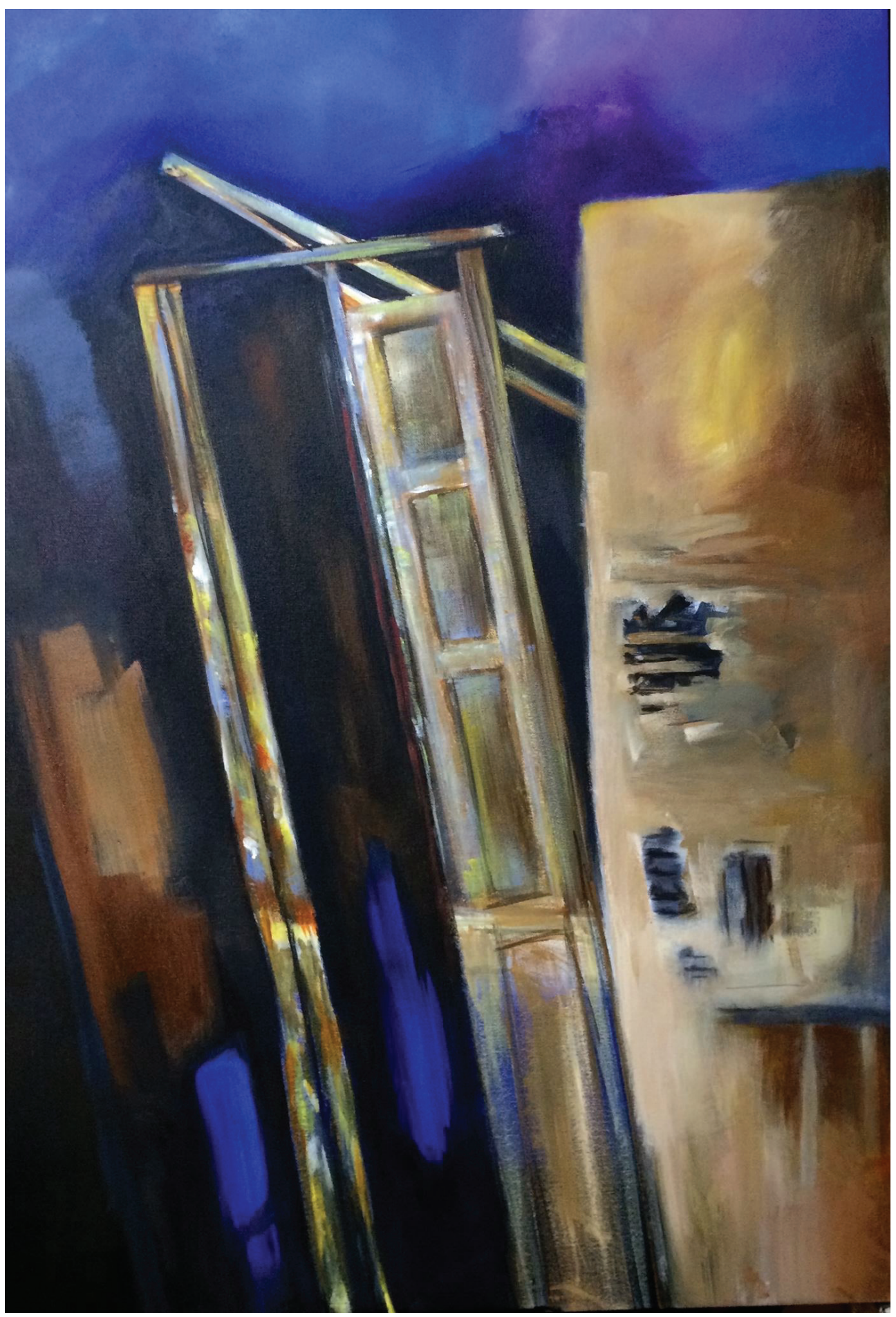

Acrílico sobre lienzo. "De la serie Puertas”, Virginia Aristizábal Parra. 